\title{
Dynamic characteristics analysis of the water storage system of the Non-supplementary Fired Compressed Air Energy Storage System
}

\author{
Ping Jiang, Ranran Chang ${ }^{a}$ and Haijian Lv
}

College of Electronic and Informational Engineering, Hebei University, Baoding 071002, China.

\author{
a1286165479@qq.com
}

\begin{abstract}
Non-supplementary Fired Compressed Air Energy Storage System (NF-CAES) consists of compressor, turbine, gas storage chamber, heat exchanger equipment, such as the complementary combustion type compressed air energy storage system is an important link in the thermal efficiency of heat exchanger. Tube heat exchanger as the research object, builds mathematical modeling. Use provided by the SIMULINK in MATLAB software components, to simulate the dynamic characteristics of the heat exchanger. According to simulation results, it is concluded that the inlet temperature of cold and hot logistics appeared different step disturbance, the change of the outlet temperature of the hot and cold logistic curve, so as to prejudge the purpose of the equipment running status and control performance.
\end{abstract}

Keywords: Non-supplementary Fired Compressed Air Energy Storage; heat exchanger; shell andtube modeling; dynamic characteristics; heat transfer; software; simulation; curve.

\section{Introduction}

In a large area of haze phenomenon under the background of energy saving and emission reduction has become a hot topic of the moment, the development of renewable energy in the world more and more attention to the large-scale development of renewable energy has become an inevitable trend under the background of global warming, but because of the wind energy and solar energy output and random fluctuations, the large-scale development of renewable energy limited, energy storage technology is the cornerstone of the large-scale use of renewable energy, can be said that the compressed air energy storage (CAES) technology is to solve the bottleneck of large-scale renewable energy development technology.

Compressed air energy storage is gradually developed in the $1950 \mathrm{~s}$, a new large-scale energy storage technology, has a large capacity of storage, the cost is relatively low, fast dynamic response, low environmental pollution, etc [1-3]. Traditional CAES system, the heat generated by the compressed air through the heat dissipation and level after cooling is lost, results in the decrease of energy storage efficiency, and to expand the compressed air in front of the need in the combustion chamber to improve the initial temperature, consumes fossil fuels and produce the waste gas, it can be seen that the traditional CAES system is a kind of energy storage hybrid generating system. Compared with the traditional system of CAES, non-supplementary fired compressed air energy storage system (NF-CAES) system has its own heat storage device [4], hot compression, storage, recovery and utilization for preheating in inflation of compressed air in the work, to improve the efficiency of the energy storage, minus the use of fossil fuels, zero emissions, truly the demand of sustainable development.

Non-supplementary Fired Compressed Air Energy Storage System(NF-CAES) this system does not need to be burning fossil fuels, and with heat storage device to recycle heat in the process of compressed air, through the heat exchanger in the stage of release can send heat to return to the turbine inlet Air, improve the thermal efficiency of the system. And heat storage of the complementary combustion type compressed air energy storage system, due to close to the adiabatic compression in the compression process, also known as advanced adiabatic compressed air energy storage system (Advanced Adiabatic Compressed Air Energy Storage, AA-CAES) [5].

The thermal device is the key to the non - combustion compressed air energy storage system, which will directly affect the performance and efficiency of the energy storage system. The NF-CAES 
system consists of energy storage and energy release stage. In energy storage phase, compressor and utilization of wind energy, light energy, the excess electricity generated by fossil fuels, such as the atmospheric pressure of compressed air into the high temperature and high pressure air, the air with a large number of compression heat, through the air in high temperature and high pressure heat exchanger with water as working medium, the compression to the cold water, thermal transfer into hot water and then into the hot water tank, air into a high pressure air storage at room temperature to the gas chamber. In the release can stage, the high pressure gas storage chamber at room temperature with compressed air into the heat exchanger heat of hot water heat exchanger, make it into a certain temperature of the air, into the expansion unit, conducive to expanding power, improve the efficiency of the expansion, the cold water to cold water tank, can be recycled.

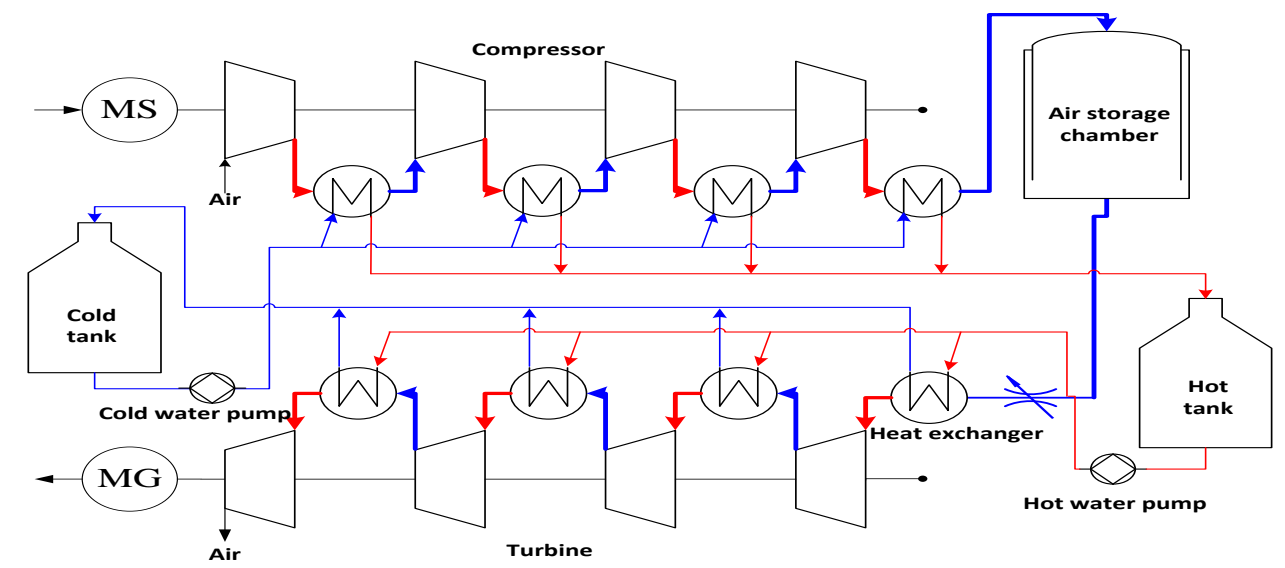

Fig. 1 Schematic diagram of non complementary combustion compressed air energy storage system

\section{NF-CAES system design parameters}

Figure 1 is a class four grade four Non-supplementary Fired Compressed Air Energy Storage System principle diagram expansion, the use of water as a heat transfer medium, the NF-CAES system usually has two storage tank, a cold water storage tank and a hot water storage tank, cold water in the water storage tank through the heat exchanger to absorb heat pump and hot water storage tank. The hot water in the hot water tank is heated by the heat exchanger to heat the air in the turbine inlet air, thereby achieving the heat return of the compression process to the turbine, increasing the output power of the turbine and improving the efficiency of the system.

As shown in Figure 1, the assumption that the compression of the NF-CAES system, the expansion of the system is a model of the four. The model of NF-CAES system using heat exchanger storage of compressed air, the cooling water as the heat storage medium, cooling after high pressure air in the gas storage chamber; storage heat exchanger heat for electricity generation in the process of heating, high pressure air in the turbine inlet.

The main assumptions of this paper are as follows:

(1) the air is an ideal gas, which satisfies the ideal gas state equation. The specific

heat capacity of air and water is determined;

(2) without considering the pressure loss in the pipeline, gas storage room and the heat exchanger, the heat loss of the heat exchanger and the heat exchanger are neglected;

(3) the compression and expansion process of heat insulation, gas storage room and the outside world for full.

\section{Transfer function of heat exchanger and establishment of simulation model}

The calculation of shell and tube heat exchanger is a complicated process, which is currently used in many ways. It is a logarithmic mean temperature difference method and NTU method. A general calculation method of shell and tube heat exchanger without phase change is proposed. The method is based on the design calculation method of heat transfer and pressure drop criterion $[5,6]$. 
Aiming at the air - water heat exchanger design and research, the design parameters were as follows: from $100.20^{\circ} \mathrm{C}$ to $32.47^{\circ} \mathrm{C}$ air cooling, cooling water inlet and outlet temperature were $39.32^{\circ} \mathrm{C}$ and $108.57^{\circ} \mathrm{C}$, the air side working pressure is $0.1 \mathrm{MPa}$, the water side pressure of $0.3 \mathrm{MPa}$.

To establish the transfer function to do the following assumptions:

(1) due to the heat transfer coefficient $k_{1}$ and $k_{2}$, the heat transfer coefficient is small and can be neglected.

(2) the heat exchange between the hot fluid, the wall surface, and the mean temperature of the cold fluid.

(3) the transfer function is derived to calculate the shell and tube heat exchanger as the analysis object.

In the process of dynamic simulation of the heat exchanger, when $\tau \leq \tau_{0}, x(\tau)=0$; when $\tau \geq \tau_{0}$, $x(\tau)=x_{0}$. In the first step a measured output disturbance after the change over time of the curve is called the step response curve or transition curve [7-9].

The general formula for the differential equation of a linear constant system:

$a_{n} \frac{d^{n} \bullet c(t)}{d \bullet t^{n}}+a_{n-1} \frac{d^{n-1} \bullet c(t)}{d \bullet t^{n-1}}+\ldots+a_{1} \frac{d \bullet c(t)}{d \bullet t}+a_{0} \bullet c(t)=b_{m} \frac{d^{m} \bullet r(t)}{d \bullet t^{m}}+b_{m-1} \frac{d^{m-1} \bullet r(t)}{d \bullet t^{m}}+\ldots+b_{1} \frac{d \bullet r(t)}{d \bullet t}+b_{0} \bullet r(t) \quad(n \geq m)$

type (1), $c(t)$ is the output variable; $r(t)$ is the input variable; $a_{n}, a_{n-1}, a_{1}$ and $a_{0}$ are constant. The initial condition of the system is zero, and the type (1) takes the Laplace transform:

$\left(a_{n} \bullet s^{n}+a_{n-1} \bullet s^{n-1}+\ldots+a_{1} \bullet s+a_{0}\right) \bullet C(s)=\left(b_{m} \bullet s^{m}+b_{m-1} \bullet s^{m-1}+\ldots+b_{1} \bullet s+b_{0}\right) \bullet R(s)$

The system transfer function is:

$G(s)=\frac{C(s)}{R(s)}=\frac{b_{m} \bullet s^{m}+b_{m-1} \bullet s^{m-1}+\ldots+b_{1} \bullet s+b_{0}}{a_{n} \bullet s^{n}+a_{n-1} \bullet s^{n-1}+\ldots+a_{1} \bullet s+a_{0}}$

Type (2), $C(s)=L[c(t)] ; R(s)=L[r(t)] ; s$ operator symbol for Laplace transform

Based on the above assumptions, the equations of the flow tube element mass conservation equation, energy conservation equation, momentum conservation equation, the equation of state and the mass, we can describe the dynamic characteristics of heat exchanger.

The micro body satisfies the following equation:

$G_{1} c_{p 1}\left(T_{1}^{\prime}-T_{1}\right)=k_{1} A_{1}\left(T_{h p}-\frac{T_{1}^{\prime}+T_{1}}{2}\right)$

$G_{2} c_{p 2}\left(T_{2}-T_{2}^{\prime}\right)=k_{2} A_{2}\left(\frac{T_{2}+T_{2}}{2}-T_{h p}\right)$

$\int p c_{p 2} \frac{d T_{h}}{d t}=k_{2} A_{2}\left(\frac{T_{2}+T_{2}^{\prime}}{2}-T_{h p}\right)-k_{1} A_{1}\left(T_{h p}-\frac{T_{1}^{\prime}+T_{1}}{2}\right)$

type (6), $G_{1}$ for water flow, $\mathrm{kg} / \mathrm{s} ; G_{2}$ for air flow, $\mathrm{kg} / \mathrm{s}$;

$T_{1}, T_{2}, T_{1}^{\prime}, T_{2}^{\prime}$ for water, air inlet and outlet temperature, $\mathrm{K}$;

$A_{1}, A_{2}$ for the water side, air side of the heat exchange area, $m^{2}$;

$T_{h p}$ is the average temperature of the metal wall of the heat exchanger,K.

Incremental equation to eliminate the intermediate variable $T_{h p}$ and $T_{2}^{\prime}$, get the relationship between $T_{1}$ and $T_{2}$ :

$$
T_{1}^{\prime}=\frac{k_{1} A_{1}}{G_{1} c_{p 1}+\frac{k_{1} A_{1}}{2}} \bullet T_{h p}+\frac{G_{1} c_{p 1}-\frac{k_{1} A_{1}}{2}}{G_{1} c_{p 1}+\frac{k_{1} A_{1}}{2}} \bullet T_{2}
$$


$T_{2}^{\prime}=\frac{k_{2} A_{2}}{G_{2} c_{p 2}+\frac{k_{2} A_{2}}{2}} \bullet T_{h p}+\frac{G_{2} c_{p 2}-\frac{k_{2} A_{2}}{2}}{G_{2} c_{p 2}+\frac{k_{2} A_{2}}{2}} \bullet T_{4}$

For conventional heat exchangers, the approximate $k_{1} A_{1} \approx k_{2} A_{2} ; G_{1} c_{p 1} \approx G_{2} c_{p 2}$.

type (7), type (8) can be written:

$T_{1}^{\prime}=\frac{2 \frac{k_{1} A_{1}}{G_{1} c_{p 1}}}{2+\frac{k_{1} A_{1}}{G_{1} c_{p 1}}} \bullet T_{h p}+\frac{2-2 \frac{k_{1} A_{1}}{G_{1} c_{p 1}}}{2+\frac{k_{1} A_{1}}{G_{1} c_{p 1}}} \bullet T_{1}$

$T_{2}^{\prime}=\frac{2 \frac{k_{2} A_{2}}{G_{2} c_{p 2}}}{2+\frac{k_{2} A_{2}}{G_{2} c_{p 2}}} \bullet T_{h p}+\frac{2-2 \frac{k_{2} A_{2}}{G_{2} c_{p 2}}}{2+\frac{k_{2} A_{2}}{G_{2} c_{p 2}}} \bullet T_{2}$

If so $\frac{k_{1} A_{1}}{G_{1} c_{p 1}}=b_{1}, \frac{k_{2} A_{2}}{G_{2} c_{p 2}}=b_{2}$ the type (9) type (10) can be simplified as:

$T_{1}^{\prime}=\frac{2 b_{1}}{2+b_{1}} \bullet T_{h p}+\frac{2-2 b_{1}}{2+b_{1}} \bullet T_{1}$

$T_{2}^{\prime}=\frac{2 b_{2}}{2+b_{2}} \bullet T_{h p}+\frac{2-2 b_{2}}{2+b_{2}} \bullet T_{2}$

The type (11), (12) into the basic type (6), and in incremental form:

$\int p c_{p 2} \frac{d T_{h}}{d t}=\frac{2 k_{2} A_{2}}{2+b_{2}}\left(\Delta T_{2}-\Delta T_{h p}\right)+\frac{2 k_{1} A_{1}}{2+b_{1}}\left(\Delta T_{1}-\Delta T_{h p}\right)$

The type (13) for the Laplace transform can be:

$$
\overline{T_{h p}}=\frac{\frac{2}{2+b_{1}}}{\frac{p c_{p 1}}{k_{1} A_{1}} \bullet s+\frac{4}{2+b_{1}}} \bullet \bar{T}_{1}+\frac{\frac{2}{2+b_{2}}}{\frac{p c_{p 2}}{k_{2} A_{2}} \bullet s+\frac{4}{2+b_{2}}} \bullet \bar{T}_{2}
$$

type (14), $\overline{T_{h p}}, \overline{T_{1}}, \overline{T_{2}}$ for the corresponding variable Laplace transform, $s$ for the Laplace transform operator.

Laplace transformation can be carried out on the $T_{1}^{\prime}, T_{2}^{\prime}$ :

$$
\begin{aligned}
& \overline{T_{1}^{\prime}}=\frac{2 b_{1}}{2+b_{1}} \overline{T_{h p}}+\frac{2-b_{1}}{2+b_{1}} \bar{T}_{1} \\
& \overline{T_{2}^{\prime}}=\frac{2 b_{2}}{2+b_{2}} \overline{T_{h p}}+\frac{2-b_{2}}{2+b_{2}} \overline{T_{2}}
\end{aligned}
$$

The type (14) on the type (15), (16), if only considering the influence of inlet temperature of water on the air outlet temperature, the influence of water inlet temperature outlet temperature of air transfer function:

$$
H_{1}(s)=\frac{2 b_{1}}{2+b_{1}} \cdot \frac{2}{\frac{p c_{p 1}}{k_{1} A_{1}} s\left(2+b_{1}\right)+4}
$$

In the same way, the transfer function of air temperature to the temperature of the outlet is affected: 


$$
H_{2}(s)=\frac{2 b_{2}}{2+b_{2}} \cdot \frac{2}{\frac{p c_{p 2}}{k_{2} A_{2}} s\left(2+b_{2}\right)+4}+\frac{2-b_{2}}{2+b_{2}}
$$

Thermodynamic calculation of shell and tube heat exchanger, constant pressure specific heat of water is $4.179 \mathrm{~J} /(\mathrm{kg} \bullet \mathrm{K})$, constant pressure specific heat of air is $1.017 \mathrm{~J} /(\mathrm{kg} \bullet \mathrm{K})$.

Can get: $b_{1}=b_{2}=0.759 ; \frac{p c_{p 2}}{k_{2} A_{2}}=261.5 ; H_{1}(s)=\frac{0.255}{721 s+1}$

\section{Implementation of simulation and simulation}

SIMULINK software provides users with a common platform for simulation and modeling. As an important subsidiary component of SIMULINK software, SIMULINK has the characteristics of accurate and fast. It can be used to build a dynamic system of heat exchanger model, is a powerful simulation tool. According to the transfer function, combined with the SIMULINK tool library, when the hot fluid inlet temperature appears step by step, the simulation program of the temperature of the outlet temperature of the cooling fluid is affected. As shown in figure (2).

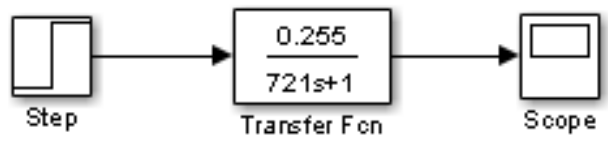

Fig. 2 Simulation of cooling fluid outlet temperature

(thermal fluid inlet temperature disturbance)

In the MATLAB running this program block diagram, get the cold logistics inlet temperature of different disturbances, the cold logistics outlet temperature of the impact of the simulation curve, as shown in Figure 3, figure 4.

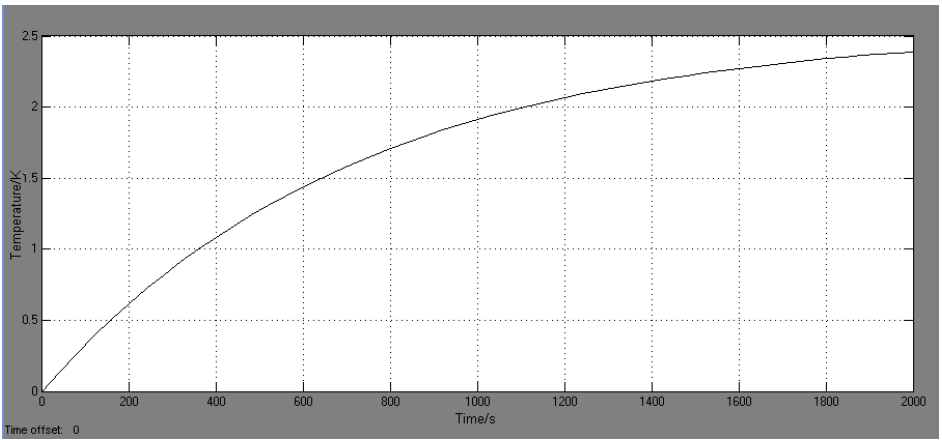

Fig. 3 Hot fluid inlet temperature rise 10K cold fluid outlet temperature simulation curve

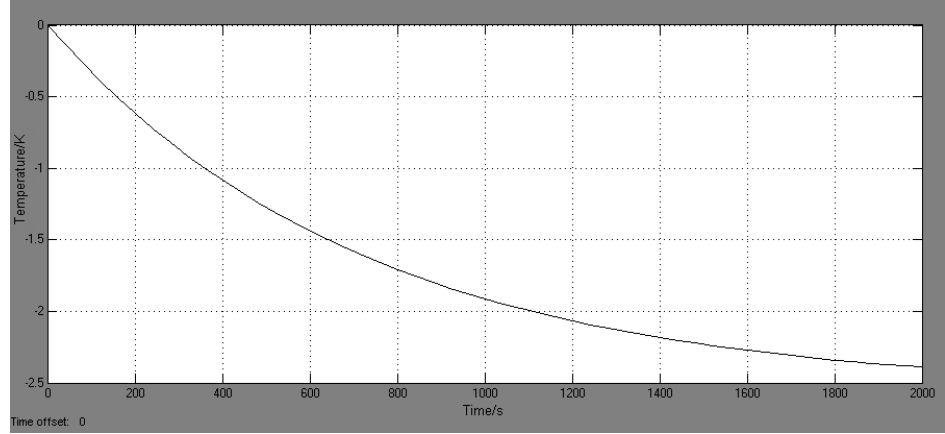

Fig. 4 Hot fluid inlet temperature drop 10K cold fluid outlet temperature simulation curve

The simulation program is shown in Figure 5, when the temperature of the inlet temperature of the cooling fluid is disturbed by the first order. 


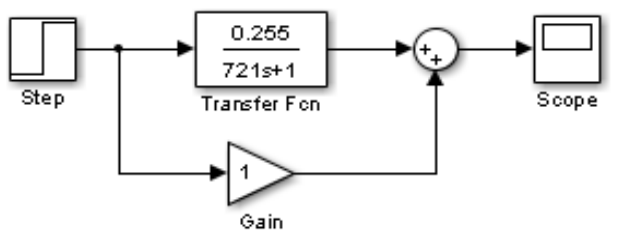

Fig. 5 Simulation of cooling fluid outlet temperature

(cooling fluid inlet temperature disturbance)

In the MATLAB run this program block diagram, get the cold logistics inlet temperature of different disturbances of the cold logistics exit temperature of the simulation curve, as shown in Figure 7, figure 6.

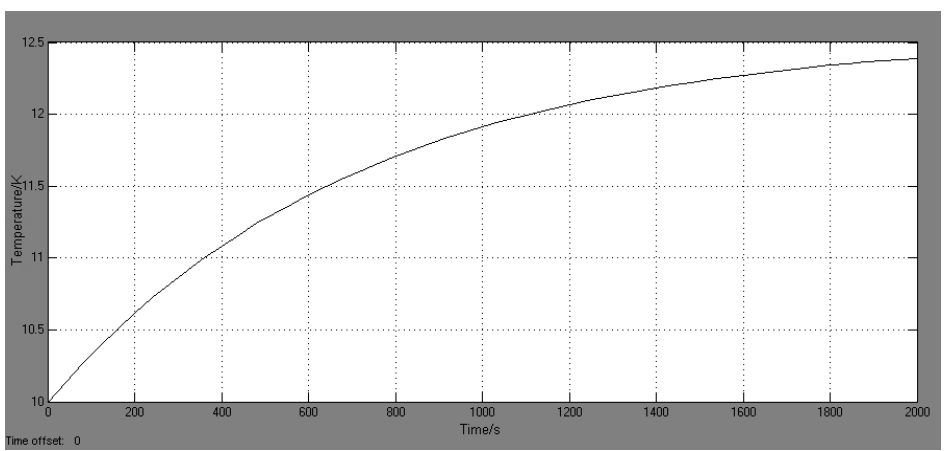

Fig. 6 Cold fluid inlet temperature rise 10K hot fluid outlet temperature simulation curve

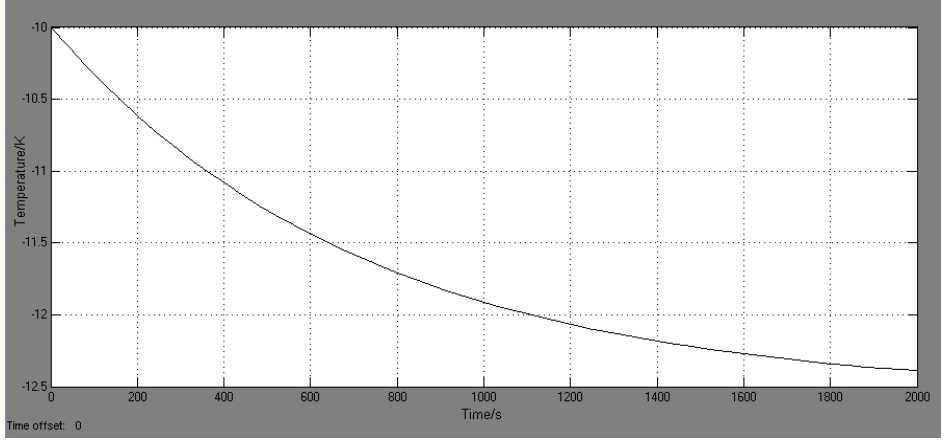

Fig. 7 Cold fluid inlet temperature drop 10K hot fluid outlet temperature simulation curve

\section{Conclusion}

Through software simulation, it is clear understanding of cold and hot fluid inlet temperature appears step perturbation, and the change of outlet temperature, the simulation result is consistent with the actual situation. According to the simulation results, the design appropriate control system, thereby reducing the delay of control process, make the control system more secure and more reliable.

\section{References}

[1] Liu Wenyi, Yang Yongping, Zhang Xiguo, et al. Compressed air energy storage (CAES) power station and its current status and development trend. Shandong electric power technology, 2007; $2 ; 10-14$.

[2] Chen H, Cong T, Yang W, et al. Progress in electrical energy storage system: a critical review. Progress in Natural Science, 2009; 19(3): 291-312.

[3] Cavallo A. Controllable and affordable utility-scale electricity from intermittent wind resources and compressed air energy storage (CAES). Energy, 2007; 32(2):120-127.

[4] Liu Jia, Yang Liang, Sheng Yong, et al. Experimental study on thermal storage of multi stage packed bed with compressed air energy storage. Power technology, 2013; 2; 1462-1468. 
[5] Yang Chonglin, Zhang Mingshi, Wang Zhongzheng. Engineering Design Manual of shell and tube heat exchanger [M]. Beijing: Mechanical Industry Press, 1998.

[6] Wang Wang, Yang Qiangsheng. The steam condensation heat transfer in the shell and tube heat exchanger [J]. Journal of Shanghai Jiao Tong University, 1998.

[7] Han Han, Zhu Xiyan, digital simulation of automatic control system, China Electric Power Press, 1996.

[8] Cheng Xiangrong, research and Simulation of the dynamic characteristics of the heat exchanger [J]. Chemical equipment technology, 2005, 26 (2), 40-43.

[9] Wu Feng. Numerical prediction and analysis of the dynamic characteristics of a continuous helical baffles heat exchanger [J]. Power engineering, 2007, 27 (4), 573-578. 